\title{
Dispersion Model for Varying Vertical Shear in Vegetated Channels
}

\author{
S. Patil ${ }^{1}$ and V. P. Singh ${ }^{2}$
}

\begin{abstract}
A dispersion model for a wide range of depthwise vertical shear is derived by using perturbation analysis and power ( $m$ ) law velocity profile. For $m=1$, the velocity profile provides linear shear, whereas $m>1$ provides nonlinear shear, and for $m>20$, the velocity profile resembles the flow through emergent vegetation. The power law represented parametrically simulates well the complex shear profiles involved in emergent and submerged vegetated flows. The proposed model shows reasonable agreement with past data on vegetated flows for a wide range of nonlinear shear velocities. DOI: 10.1061/(ASCE)HY.1943-7900.0000431. (C) 2011 American Society of Civil Engineers.
\end{abstract}

CE Database subject headings: Channels; Vegetation; Dispersion; Velocity; Shear.

Author keywords: Dispersion; Perturbation; Shear velocity.

\section{Introduction}

When pollutants in turbulent flow are transported downstream, vertical and transverse velocity profiles and cross-sectional mixing spread them over the cross section of flow, after which the balance between longitudinal shear and cross-sectional diffusion sets up longitudinal shear dispersion. Several empirical expressions have been proposed for the longitudinal dispersion coefficient for specific geometric channel configurations (Liu 1977; Seo and Cheong 1998; Deng et al. 2002; Kashefipour and Falconer 2002; Seo and Baek 2004). Elder (1958) proposed a theoretical expression for a wide-open channel as

$$
K=-\frac{1}{d} \int_{0}^{h} \tilde{U}(y) \int_{0}^{y} \frac{1}{e_{y}} \int_{0}^{y} \tilde{U}(y) d y d y d y=\frac{I}{e_{y}}
$$

in which $K=$ longitudinal dispersion coefficient, $d=$ flow depth, $e_{y}=$ vertical diffusivity, and $y=$ depthwise dimension. $\tilde{U}(y)=$ $\bar{U}-U(y)=$ shear, in which $\bar{U}=$ depthwise average velocity and $U(y)=$ depthwise profile of flow velocity. Taylor (1954) and Fischer (1968) have derived similar expressions for flow through pipes and natural streams, respectively. Eq. (1) suggests that the dispersion coefficient is precisely governed by the relative magnitude of shear in the velocity profile and diffusivity, and thus one can calculate the accurate magnitude of the dispersion coefficient if the velocity profile and diffusivity are known. Fischer (1968) showed that dispersion owing to the transverse variation of streamwise velocity is much greater than the vertical

${ }^{1}$ Dept. of Biological and Agricultural Engineering, Texas A \& M Univ., 2117 TAMU, College Station, TX 77843-2117 (corresponding author). E-mail: ceenrne@ymail.com

${ }^{2}$ Caroline and William N. Lehrer Distinguished Chair in Water Engineering and Professor, Dept. of Biological and Agricultural Engineering and Dept. of Civil and Environmental Engineering, Texas A \& M Univ., 2117 TAMU, College Station, TX 77843-2117.

Note. This manuscript was submitted on July 23, 2008; approved on March 29, 2011; published online on September 15, 2011. Discussion period open until March 1, 2012; separate discussions must be submitted for individual papers. This technical note is part of the Journal of Hydraulic Engineering, Vol. 137, No. 10, October 1, 2011. CASCE, ISSN 07339429/2011/10-1293-1297/\$25.00. variation (Fischer et al. 1979). The term $e_{y}$ is often assumed to be constant because its magnitude can be easily calculated from the bed shear velocity. However, the contribution from $U^{\prime}(y)$ [included in the term $I$ in Eq. (1)] involves numerous calculations that make Eq. (1) difficult to solve. Moreover, any change in the vertical velocity profile may potentially alter the magnitude of $K$. To assess this change, Eq. (1) must be solved all the way from the beginning for every change in the velocity profile. This intricacy motivated us to derive a general result for a range of velocity profiles in this note. The derivation uses power law (Vennard 1961; Kundu and Cohen 2002; Cheng 2007) to calculate velocity shear. Power law, because it performs better than log law (Hinze 1975; Cheng 2007), has been popularly used in smooth pipe flows (Schlichting 1979) and open channel flows (Karim and Kennedy 1987; Bergstrom et al. 2001; Cheng 2007). For most of these flows, the power law has been shown to fit well for a range of the $m$ values. Studies on open-channel flows show a range of 4-12 m values for different Reynolds numbers (Chen 1991; Qian and Wan, 1999). Chen (1991) suggested a general value of $m \sim 6$ for the usual practical flows, whereas Smart et al. (2002) suggested reduction up to $m \sim 2$ for flows with larger scale bed roughness (Nowell and Church 1979). Odgaard and Bergs (1988) used the power law to model velocity distribution in a curved channel, whereas Odgaard (1981) extended Falcon's (1979) analysis based on the power law to propose an improved model for steady-state transverse bed profile.

In this paper, we used the power law velocity profile, represented in a parametric form, to calculate shear in vegetated channels as a function of power; following perturbation analysis, we derived an expression for longitudinal dispersion coefficient as a function of power. The change in the $m$ value in the power law velocity provides a corresponding change in the vertical velocity profile, which in turn changes the shear. The proposed closed-form expression for dispersion coefficient calculates the corresponding change in the magnitude of longitudinal dispersion, thus eliminating the repetitive calculations required in Eq. (1). Multiple time scales have been used for convection and diffusion processes. The proposed derivation is limited to the vertical shear; i.e., it follows Elder's (1958) assumption of an infinitely wide-open channel with no variation in transverse flow velocity. 


\section{Perturbation Analysis}

We can estimate the scales required to conduct perturbation analysis using the vertical profile of flow velocity $(U)$ as $U=U(y)$ in $0<y<d$, in which $d$ is the flow depth and $y$ is the vertical coordinate. The two-dimensional mass transport equation for the pollutant concentration $C$ can be written as

$$
\frac{\partial C}{\partial t}+\frac{\partial U C}{\partial x}=D\left(\frac{\partial^{2} C}{\partial x^{2}}+\frac{\partial^{2} C}{\partial y^{2}}\right)
$$

with no flux boundary conditions $\partial C / \partial y=0$ at $y=0$ and $d$. In Eq. (2), $C(x, y, t)=$ concentration of pollutants, $U=$ depthwise vertical velocity profile, $D=$ turbulent diffusion coefficient, and $x$ and $y=$ horizontal and vertical dimensions, respectively. The solution of Eq. (2) depends on two time scales: the horizontal advection time $T_{a}=L / U_{m}$, in which $U_{m}$ is the horizontal velocity scale, and the vertical diffusion time scale is $T_{d}=d^{2} / D$. A parameter $\varepsilon \ll 1$ is used to represent the ratio of these two time scales as $L / U_{m}: L^{2} / D=1 / \varepsilon: 1 / \varepsilon^{2}$, in which $U_{m}$ is the maximum horizontal velocity and $L$ is the longitudinal scale such that $d / L \ll 1$. Assuming that $\varepsilon \ll 1$ and $P=d U_{m} / D=O(1)$, the dimensionless variables $x=L x^{\prime} ; y=d y^{\prime} ; U=U_{m} U^{\prime} ; t=\left(L / U_{m}\right) t^{\prime} ;$ and $C=$ $C_{m} C^{\prime}$ with vertical diffusion time scale are used in Eq. (2) to yield

$$
P \varepsilon\left[\frac{\partial C^{\prime}}{\partial t^{\prime}}+\frac{\partial U^{\prime} C^{\prime}}{\partial x^{\prime}}\right]=\varepsilon^{2} \frac{\partial^{2} C^{\prime}}{\partial x^{\prime 2}}+\frac{\partial^{2} C^{\prime}}{\partial y^{\prime 2}}
$$

with boundary conditions becoming $\partial C^{\prime} / \partial y^{\prime}=0$ at $y^{\prime}=0$ and $y^{\prime}=1$. For convenience, primes on the variables are henceforth dropped out. The solution of Eq. (3) has two independent time scales, $t_{0}=t$ and $t_{1}=\varepsilon t$. Following the chain rule, the time derivative in Eq. (3) can be written as

$$
\frac{\partial C}{\partial t} \rightarrow \frac{\partial C}{\partial t_{0}} \frac{\partial t_{0}}{\partial t}+\frac{\partial C}{\partial t_{1}} \frac{\partial t_{1}}{\partial t}=\frac{\partial C}{\partial t_{0}}(1)+\frac{\partial C}{\partial t_{1}}(\varepsilon)
$$

Introducing multiple perturbation for the concentration as $C=$ $C_{0}+\varepsilon C_{1}+\varepsilon^{2} C_{2}$ with $C_{i}=C_{i}\left(x, y, t_{0}, t_{1}\right)$ for all $i=1,2,3, \ldots$ and substituting Eq. (4) in Eq. (3), the perturbation problems of first and second order in $\varepsilon$ are

$$
\begin{gathered}
O\left(\varepsilon^{1}\right): P\left[\frac{\partial C_{0}}{\partial t_{0}}+\frac{\partial U C_{0}}{\partial x}\right]=\frac{\partial^{2} C_{1}}{\partial y^{2}}, \frac{\partial C_{1}}{\partial y}=0 \quad \text { at } y=0,1 \\
O\left(\varepsilon^{2}\right): P\left[\frac{\partial C_{1}}{\partial t_{0}}+\frac{\partial C_{0}}{\partial t_{1}}+\frac{\partial U C_{1}}{\partial x}\right]=\frac{\partial^{2} C_{0}}{\partial x^{2}}+\frac{\partial^{2} C_{2}}{\partial y^{2}}, \\
\frac{\partial C_{2}}{\partial y}=0 \quad \text { at } y=0,1
\end{gathered}
$$

Assuming unit width of a channel, we can find concentration $C_{0}$ by averaging Eq. (5) across the channel depth and involving no flux boundary condition

$$
\frac{1}{d} \int_{0}^{d} P\left[\frac{\partial C_{0}}{\partial t_{0}}+\frac{\partial U C_{0}}{\partial x}\right] d y=\frac{1}{d} \int_{0}^{d} \frac{\partial^{2} C_{1}}{\partial y^{2}} d y
$$

The solution to Eq. (7) is given by the solution to $\partial C_{0} / \partial t_{0}+\bar{U}\left(\partial C_{0} / \partial x\right)=0$. Multiplying by $P$ and subtracting the result from Eq. (6) leads to

$$
P(\tilde{U}) \frac{\partial C_{0}}{\partial t_{0}}=\frac{\partial^{2} C_{1}}{\partial y^{2}}
$$

in which $\tilde{U}=U-\bar{U}=$ velocity defect. The solution for Eq. (8) is of the form $C_{1}=P\left(\partial C_{0} / \partial t_{0}\right) B(y)$. Substituting in Eq. (8),
$\tilde{U}=\partial^{2} B(y) / \partial y^{2} \quad$ with boundary conditions $\partial C_{1} / \partial y=0$; $\partial B(y) / \partial y=0$ at $y=0,1$. The term $P \partial U C_{1} / \partial x$ in Eq. (7) can be reformulated using $\tilde{U}$ as $P\left(\partial U C_{1} / \partial x\right)=P(\tilde{U}+\bar{U})\{(\partial / \partial x)$ $\left.\left[P\left(\partial C_{0} / \partial x\right) B(y)\right]\right\}=P^{2}(\tilde{U}+\bar{U}) B(y)\left(\partial^{2} C_{0} / \partial x^{2}\right)$ and the term $P\left(\partial C_{1} / \partial t_{0}\right) \quad$ is $P\left(\partial C_{1} / \partial t_{0}\right)=P\left\{\left(\partial / \partial t_{0}\right)\right\}\left[P\left(\partial C_{0} / \partial x\right) B(y)\right]=$ $-P^{2} B(y) \bar{U}\left[\partial^{2} C_{0} / \partial x^{2}\right]$. Substituting in Eq. (6) and averaging over cross section, it is

$$
\frac{\partial C_{0}}{\partial t_{1}}=\left(1-P^{2} \overline{\tilde{U} B(y)}\right) \frac{\partial^{2} C_{0}}{\partial x^{2}}
$$

in which overbar is the depth averaged. Reverting to dimensional variables and substituting for the long time scale leads to

$$
\frac{\partial C_{0}}{\partial t}+\bar{U} \frac{\partial C_{0}}{\partial x}=K_{x} \frac{\partial^{2} C_{0}}{\partial x^{2}}
$$

in which $K_{x}=D\left(1-P^{2} \overline{\tilde{U} B(y)}\right)$ represents the longitudinal dispersion coefficient.

\section{Longitudinal Dispersion Coefficient}

We can use the power law velocity profile to represent the flow velocity, which in dimensionless form may be written as

$$
\frac{U}{U_{m}}=\left(\frac{y}{2 T_{1} d}\right)^{m}
$$

in which $m=$ power and its value depends on Reynolds number $\mathrm{R}=4 \bar{U} d / \gamma\left(\gamma=\right.$ kinematic viscosity $\left.=10^{-6} \mathrm{~m}^{2} / \mathrm{s}\right)$. We employ a usual substitution of pipe radius equaling twice the flow depth to represent the depthwise vertical velocity profile in open channels. Moreover, the location of maximum velocity $\left(U_{m}\right)$ can be represented as a fraction of flow depth, $T_{1} d$. For example, in uniform flow, the distance of $U_{m}$ is $0.9 d$ from the channel bottom. Although Eq. (11) is a monotonic function, we can use it as an approximate model for measured velocity profile, which has a maximum below the water surface. Fig. 1 shows a linear relationship between $m$ and R (Modi and Seth 1990), from which we can select the value of $m$ for a certain value of $\mathrm{R}$. For different turbulent flows, we can select different $\mathbf{R}$ and hence $m$, and we can obtain corresponding vertical shear velocity profiles. To illustrate, $U_{m}=1$ is assumed in Eq. (11)

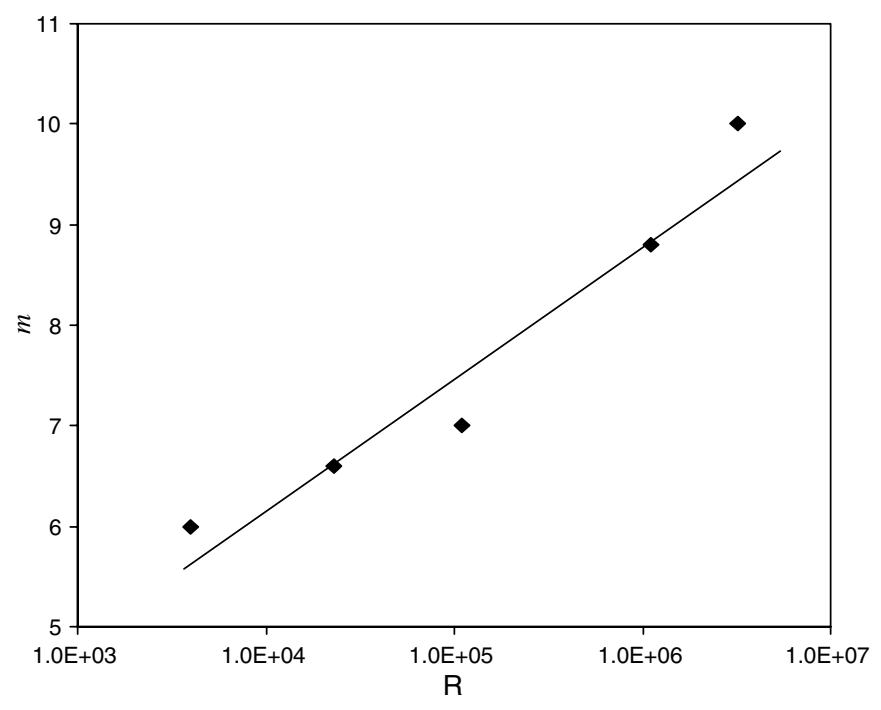

Fig. 1. Reynolds number versus power $(m)$ 


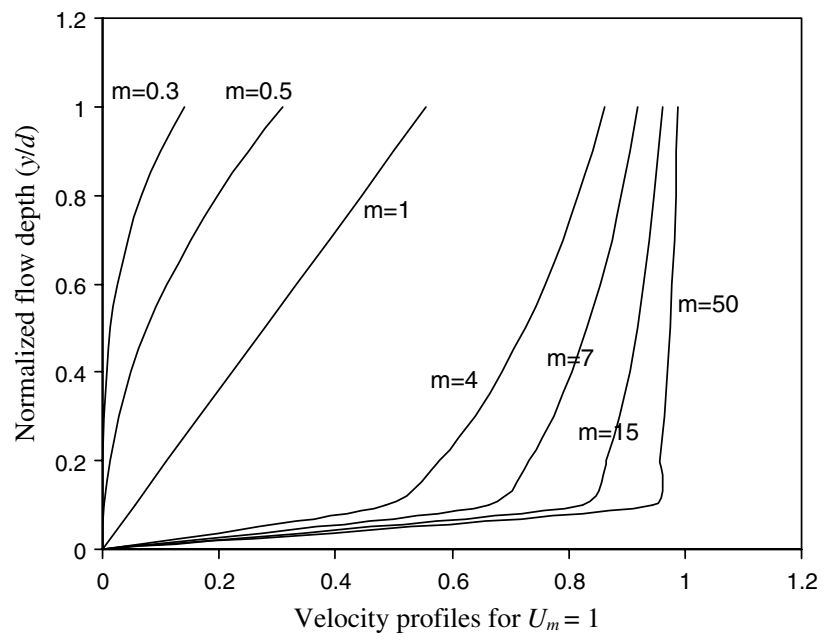

Fig. 2. Shear velocity profiles according to magnitude of $m$

and $U$ versus $y / d$ is plotted in Fig. 2 for various values of $m$. The figure shows various vertical profiles of flow velocities, which resemble one or the other velocity profile that one can observe in the field. For example, $m=1$ provides linear shear current, $m \sim 7$ provides logarithmic profile in uniform flow, and $m>15$ corresponds to the current profile in coastal areas or the velocity profile of flow through vegetation. Moreover, for $m<1$, the velocity profile reverses to become exponential and provides inverse shear velocity, which resembles that induced by a hurricane. One can find the average velocity $(\bar{U})$ over the flow depth from Eq. (11) by substituting $y=T_{2} d$, in which $T_{2}$ is the fraction of flow depth at which the average value of flow velocity lies. For example, $\bar{U}$ in open channels lies at $y=0.4 d$ from the channel bottom, wherein $T_{2}=0.4$. We can use Eq. (11) to write equation for $\bar{U}$ in dimensionless form as

$$
\frac{\bar{U}}{U_{m}}=\left(\frac{T_{2} d}{2 T_{1} d}\right)^{m}=\left(\frac{T_{2}}{2 T_{1}}\right)^{m}
$$

The shear $\tilde{U}$ from Eqs. (11) and (12) is $\tilde{U}=\left(y / 2 T_{1} d\right)^{m}-$ $\left(T_{2} / 2 T_{1}\right)^{m}$. Substituting in Eq. (8) and integrating provides

$$
\frac{\partial B(y)}{\partial y}=\frac{\left(\frac{y}{2 T_{1} d}\right)^{m+1} 2 T_{2} d}{(m+1)}-\left(\frac{T_{2}}{2 T_{1}}\right)^{m} y+B_{1}
$$

in which $B_{1}=0=$ integration constant for the boundary condition $\partial B / \partial y=0$ at $y=0$. Further integrating Eq. (13), one obtains

$$
B(y)=\frac{y^{m+2}}{(m+1)(m+2)\left(2 T_{1}\right)^{m}}-\left(\frac{T_{2}}{2 T_{1}}\right)^{m} \frac{y^{2}}{2}+B_{2}
$$

Using the condition that $\bar{B}(y)=\int_{0}^{1} B(y) d y=0$, the result is

$$
B_{2}=\left(\frac{T_{2}}{2 T_{1}}\right)^{m} \frac{1}{6}-\frac{1}{(m+1)(m+2)(m+3)\left(2 T_{1}\right)^{m}}
$$

Using Eq. (14) and $\tilde{U}$, one gets

$$
\begin{aligned}
\overline{\tilde{U} B(y)}= & \int_{0}^{1}\left\{\left(\frac{y}{2 T_{1}}\right)^{m}-\left(\frac{T_{2}}{2 T_{1}}\right)^{m}\right\} \\
& \times\left\{\frac{y^{m+2}}{(m+1)(m+2)\left(2 T_{1}\right)^{m}}-\left(\frac{T_{2}}{2 T_{1}}\right)^{m} \frac{y^{2}}{2}+\left(\frac{T_{2}}{2 T_{1}}\right)^{m} \frac{1}{6}\right. \\
& \left.-\frac{1}{(m+1)(m+2)(m+3)\left(2 T_{1}\right)^{m}}\right\} d y
\end{aligned}
$$

Simplifying Eq. (16) and substituting in the mixing coefficient

$$
\begin{aligned}
D(1 & \left.-P^{2} \overline{\tilde{U} B(y)}\right), \text { it is } \\
D & -D\left(\frac{U_{m} d}{D}\right)^{2}\left\{\frac { 1 } { ( 2 T _ { 1 } ) ^ { 2 m } } \left[\frac{1}{\xi(2 m+3)}-\frac{1}{\xi(m+1)(m+3)}\right.\right. \\
& \left.\left.-\left(\frac{T_{2}}{2 T_{1}}\right)^{m} \frac{1}{2(m+3)}+\frac{1}{6(m+1)}\right]-\left(\frac{T_{2}}{2 T_{1}}\right)^{m} \frac{2}{(m+3) \xi}\right\}
\end{aligned}
$$

in which $\xi=(m+1)(m+2)\left(2 T_{1}\right)^{m}$. In Eq. (17), the first term is the molecular diffusion and the second term is the longitudinal dispersion coefficient $(K)$ owing to shear flow, which is represented as

$$
\begin{aligned}
K= & -\frac{\left(U_{m} d\right)^{2}}{D}\left\{\frac { 1 } { ( 2 T _ { 1 } ) ^ { m } } \left[\frac{1}{\xi(2 m+3)}-\frac{\left(T_{2}\right)^{m}}{2\left(2 T_{1}\right)^{m}(m+3)}\right.\right. \\
& \left.\left.+\frac{\left(T_{2}\right)^{m}}{6\left(2 T_{1}\right)^{m}(m+1)}-\frac{1}{\xi(m+1)(m+3)}\right]\right\}
\end{aligned}
$$

Moreover, one can calculate the fraction $T_{2}$ by averaging Eq. (11) over the flow depth as $\bar{U}=(1 / d) \int_{0}^{d} U d y=U_{\max } /$ $\left[\left(2 T_{1}\right)^{m}(m+1)\right]$. From this and Eq. (12), $T_{2}=[1 /(m+1)]^{1 / m}$. The factor $2 T_{1}$ is the fraction of the flow depth from bottom where the maximum velocity lies, which can be approximately fixed, whereas one can calculate $T_{2}=f(m)$ by properly selecting $m$. Eq. (18) provides a generalized closed-form expression for the longitudinal dispersion coefficient. For a Reynolds number corresponding to a particular channel flow, one can use Fig. 1 to find the corresponding magnitude of $m$, and using Eq. (18), one can assess the longitudinal dispersion coefficient for that channel flow.

\section{Application}

Fig. 2 shows different shear velocities that exist in the field, for which one can calculate the corresponding dispersion coefficients from Eq. (18). This explains the versatility of Eq. (18). The prediction accuracy of Eq. (18) lies in the selection of $m$. For example, in a uniform flow experiment, the depthwise velocity profile should be logarithmic; however, it generally deviates in practice because of laboratory constraints. Umeyama (2005) suggested a better prediction by using a power series law in place of logarithmic law. However, the power series requires large computations; moreover, its accuracy depends on the order of the series under consideration. The present analysis simplifies the approach and allows for the prediction of dispersion coefficient simply by a suitable selection of $m$.

Experimental data of emergent (Nepf et al. 1997) and submerged (Murphy et al. 2007) vegetation have been used to examine the performance of the derived model. In emergent vegetation, Nepf et al. (1997) used a 38-cm-wide and 20-m-long flume with 1-m length of vegetation canopy having $0.6-\mathrm{cm}$-diameter wooden dowels to simulate the vegetative flow. For the mean inlet flow $=0.06 \mathrm{~m} / \mathrm{s}$, they measured the depthwise velocity profiles first in a bare-bed channel (without vegetation) and then in the presence of vegetation with varying stem densities as $1,1.5$, and $5.5 \%\left(280,430,1,700\right.$ plants $\left./ \mathrm{m}^{2}\right)$, respectively. Their data of all four velocity profiles were replotted in Fig. 3. The velocity profiles predicted by Eq. (11) are also plotted with $m$ adjusted to get the proper fit. Fig. 4 shows the observed dispersion coefficients for the preceding four cases, measured using dye experiments. This figure also shows the corresponding dispersion coefficients predicted experimentally and those predicted by Eq. (18). We see some deviations in the predicted values of Nepf et al. (1997), which have been reasoned out because of the effect similar to the dead zone 


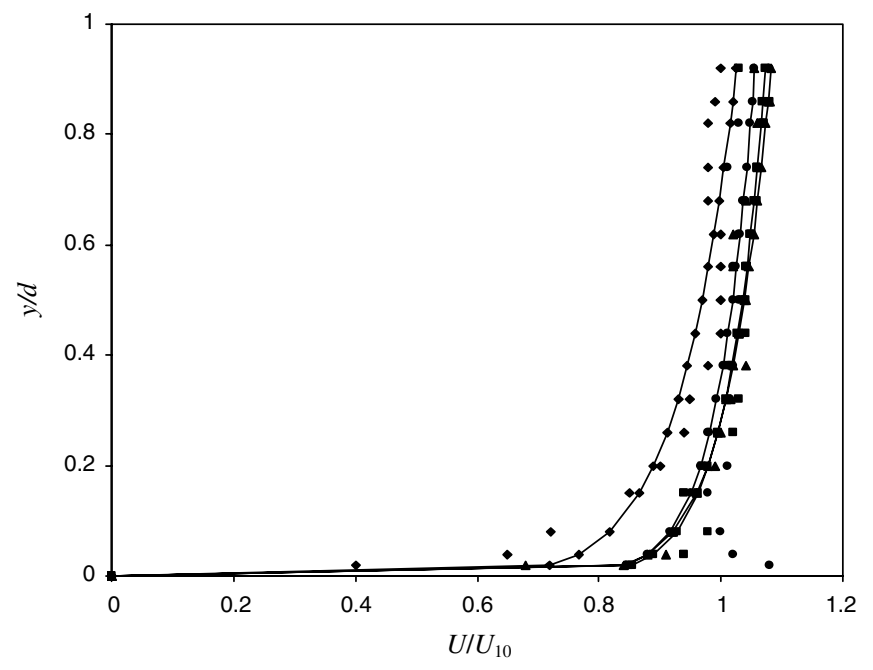

Fig. 3. Depthwise velocity profiles: observed data (dots); predicted (lines); $\downarrow$ = bare-bed; $\boldsymbol{\Delta}=1 \%$ vegetation density ( $\mathrm{vd}) ; \mathbf{\square}=1.5 \% \mathrm{vd}$; $\cdot=5.5 \% \mathrm{vd}$

(Valentine and Wood 1977; Keefe et al. 2004) exercised by vegetation, whereas the dispersion coefficients for bare-bed as well as vegetated flows are reasonably simulated by the present model. Elder's (1958) expression for the dispersion coefficient has also been used to verify dispersion in bare-bed channels because his work provides the necessary input: velocity profile (logarithmic equation) and expression for dispersion coefficient $\left(=5.93 d U_{*}\right)$. $m \sim 8$ in the power law equation provided $T 2 \sim 0.4$, at which the average velocity of uniform flow lies. The maximum velocity was calculated using $U_{m}=23 d^{0.63}$ and bed slope $=0.0008$ from a given inclined angle $0.046^{\circ}$ of flume (Elder 1958). We used this data in Eq. (18) to calculate dispersion coefficients for flow depths, for which the limit $d<1.5 \mathrm{~cm}$ given by Elder (1958). We then matched these magnitudes with Elder's expression for the dispersion coefficient $=5.93 d U_{*}$. We found reasonable matching, as shown in Fig. 4, in which deviations for $d \rightarrow 1.5 \mathrm{~cm}$ occur because the validity of Elder's model reduces toward this value.

We also used data from Ghisalberti and Nepf (2004), who measured velocity profiles in submerged vegetation. The stem height for all three runs was $13.8 \mathrm{~cm}$, whereas the stem density varied from 0.025 to $0.080 / \mathrm{cm}$ for seven runs, namely $A, C, D, E, G$, $H$, and $I$. Murphy et al. (2007) measured the corresponding dispersion coefficient values, which we reproduced in Fig. 4. To validate the derived equation, we used the velocity profiles for the preceeding runs (Fig. 5) as given in Ghisalberti and Nepf (2004) to predict the dispersion coefficient. Because of the drag exerted by stems, the flow velocity in vegetation region is much slower than the overflow velocity (Carollo et al. 2002); thus it exhibits a point of inflection at the top of vegetation. For such velocity profiles, in which the point of inflection is an important feature, constructing the velocity profile was possible by using cumulative power; i.e., $m_{c(n+1)}=m_{0}+n \Delta m+m_{c n}$, in which subscript $c$ denotes the cumulative value of $m$ and $n$ denotes the steps that correspond to the data points of $y$ in $y / d$. With the initial $m_{0} \sim 0$ at the surface (finite value $\ll 1$ was taken for practical purposes as $T_{2}=\infty$ at $m=0$ ), we predicted the velocity profile by selecting a proper value of the incremental power, $\Delta m$. Thus, the cumulative magnitude of power eventually provides initiation with $m \sim m_{0}$ at the surface and ends at the bottom for $m>1$, with the point of inflection at around $m \sim 1$. Thus, from the surface to the point of inflection, the concave velocity profile was simulated by $0<m<1$, and for the convex profile from the inflection point to the bottom, it was

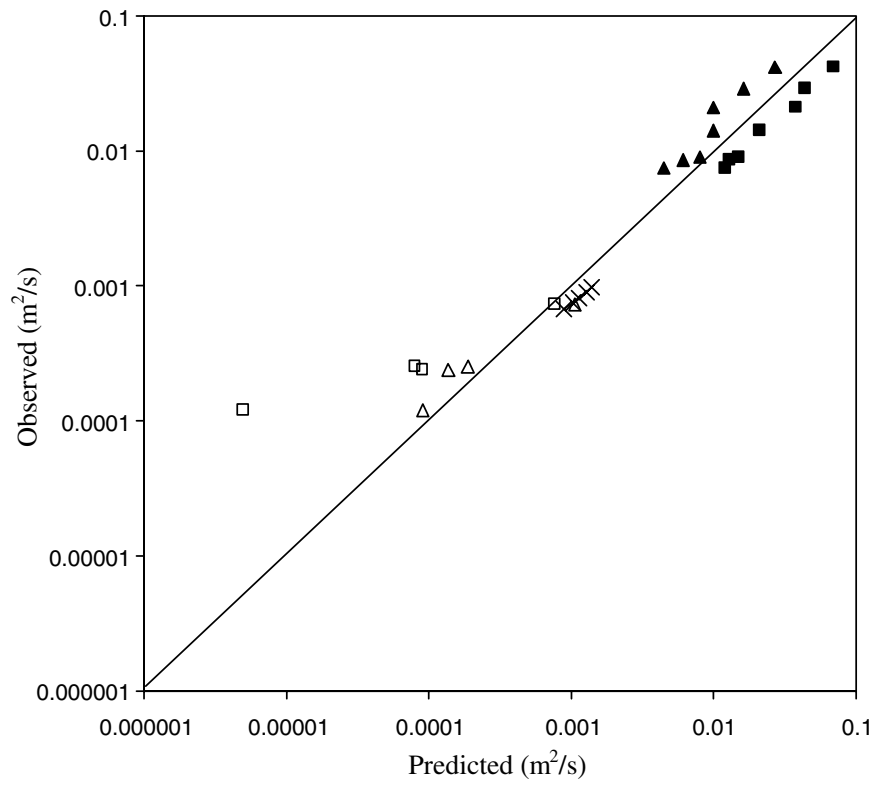

Fig. 4. Predicted versus observed dispersion coefficients for emergent (open points) and submerged (dark points) vegetation. $\mathbf{~ = ~ p a s t ~ a u t h o r s ; ~}$ $\boldsymbol{\Delta}=$ present theory; $\mathrm{x}=$ Elder's data for bare-bed channel

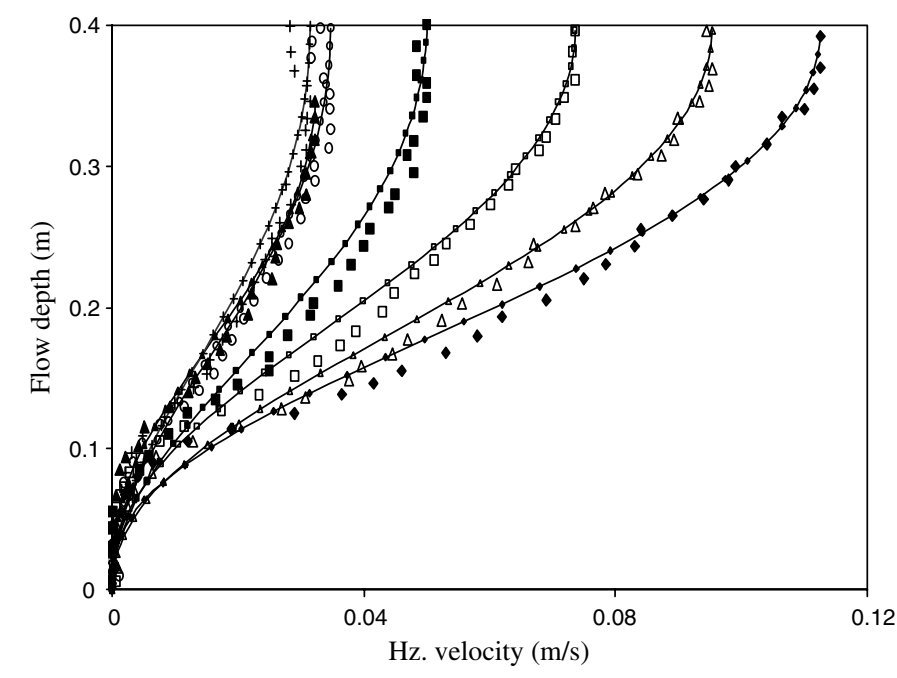

Fig. 5. Depthwise velocity profiles measured (dots); predicted (lines); $\Delta=\operatorname{run} G ; \boldsymbol{\nabla}=\operatorname{run} C ; \diamond=\operatorname{run} H$

simulated by $m>1$. The cumulative law previously described properly provides the necessary increase in $m$ to simulate the entire profile. For every $y / d$, the corresponding cumulative value of $m$ was assigned. The constructed velocity profile was solved by the perturbation method, previously described, to get the dispersion coefficient. We calculated the vertical diffusivity $D=0.013\left(\Delta U t_{m l}\right)$ (in which $\Delta U$ is the difference between overflow and vegetation velocity and $t_{m l}$ is the thickness of mixing layer) as suggested in Murphy et al. (2007). We plotted the dispersion coefficients for the seven runs in Fig. 4, which shows good predictions by the derived equation with the observed data of Murphy et al. (2007).

\section{Conclusion}

This paper proposes a generalized expression for the longitudinal dispersion coefficient for vertical shear that provides its magnitude 
according to the change in the shear velocity. The power law is used to simulate flow velocity, in which the power term is maintained as variable, and perturbation analysis to the second order is conducted to obtain a closed-form solution of the dispersion coefficient. The proposed expression has the capability to adapt to a wide range of shear velocity profiles from linear to nonlinear, including common velocities such as logarithmic profile in uniform flow, vertically straight profile existing in coastal regions, and so on. In case of $m<1$, the power law also simulates the velocity pattern of a hurricane. The obtained closed-form equation is applied to simulate the observed dispersion coefficients in the bare-bed flow, flow through emergent vegetation, and submerged vegetation. Results show the applicability of the proposed generalized equation for dispersion in vertical shear flow, and may be a useful contribution to the wetland mechanics (Carleton and Montas 2010; Vandenbruwaene et al. 2011). In view of the numerous individual dispersion coefficients proposed in the past for particular sitespecific conditions, the present expression can be considered a more general expression that includes most of the depthwise velocity patterns observed in the field.

\section{References}

Bergstrom, D. J., Tachie, M. F., and Balachandar, R. (2001). “Application of power law to low Reynolds number boundary layers on smooth and rough surfaces." Phys. Fluids, 13(11), 3277-3284.

Carleton, J. N., and Montas, H. J. (2010). "An analysis of performance models for free water surface wetlands." Water Res., 44(12), 3595-3606.

Carollo, F., Ferro, V., and Termini, D. (2002). "Flow velocity measurements in vegetated channels." J. Hydraul. Eng., 128(7), 664-673.

Chen, C. I. (1991). "Unified theory on power laws for flow resistance." J. Hydraul. Eng., 117(3), 371-389.

Cheng, N. S. (2007). "Power-law index for velocity profiles in open channel flows." Adv. Water Resour., 30, 1775-1784.

Deng, Z. Q., Bengtsson, L., Singh, V. P., and Adrian, D. D. (2002). "Longitudinal dispersion coefficient in single-channel streams." J. Hydraul. Eng., 128(10), 901-916.

Elder, J. W. (2006). "The dispersion of marked fluid in turbulent shear flow." J. Fluid Mech., 5, 544-560.

Falcon, M. A. (1979). "Analysis of flow in alluvial channel bends." Ph.D. thesis, University of Iowa, Iowa City, IA.

Fischer, H. B. (1968). "Dispersion predictions in natural streams." J. Sanit. Eng. Div., 94(5), 927-944.

Fischer, H. B., List, E. J., Koh, R., Imberger, J., and Brooks, N. (1979). Mixing in inland and coastal waters, Academic, New York, 483.

Ghisalberti, M., and Nepf, H. (2004). "The limited growth of vegetated shear layers." Water Resour. Res., 40, 1-12.
Hinze, J. O. (1975). Turbulence, 2nd Ed., Vol. X, McGraw-Hill, New York, 790.

Karim, M. F., and Kennedy, J. F. (1987). "Velocity and sedimentconcentration profiles in river flows." J. Hydraul. Eng., 113(2), $159-178$.

Kashefipour, S. M., and Falconer, R. A. (2002). "Longitudinal dispersion coefficients in natural channels." Water Res., 36(6), 1596-1608.

Keefe, S. H., Barber, L. B., Runkel, R. L., Ryan, J. N., McKnight, D. M., and Wass, R. D. (2004). "Conservative and reactive solute transport in constructed wetlands." Water Resour. Res., 40, 1-12.

Kundu, P. K., and Cohen, I. M. (2002). Fluid mechanics, Academic, San Diego, CA, 730.

Liu, H. (1977). "Predicting dispersion coefficient of stream." J. Envir. Eng. Div., 103(1), 59-69.

Modi, M., and Seth, H. (1990). Fluid mechanics and hydraulics, Khanna, New Delhi, India.

Murphy, E., Ghisalberti, M., and Nepf, H. (2007). "Model and laboratory study of dispersion in flows with submerged vegetation." Water Resour. Res., 43, 1-12.

Nepf, H., Mugnier, C., and Zavistoski, R. (1997). "The effects of vegetation on longitudinal dispersion." Estuarine, Coastal Shelf Sci., 44(6), 675-684.

Nowell, A. R.M., and Church, M. (1979). "Turbulent flow in a depthlimited boundary layer.” J. Geophys. Res., 84(C8), 4816-4824.

Odgaard, A. J. (1981). "Transverse bed slope in alluvial channel bends." J. Hydraul. Div., 107(12), 1677-1694.

Odgaard, A. J., and Bergs, M. A. (1988). "Flow processes in a curved alluvial channel." Water Resour. Res., 24(1), 45-56.

Qian, N., and Wan, Z. (1999). Mechanics of sediment transport, Vol. XIX, ASCE, Reston, VA, 913.

Schlichting, H. (1979). Boundary-layer theory, 7th Ed., Vol. XXII, McGraw-Hill, New York, 817.

Seo, I. W., and Baek, K. O. (2004). "Estimation of the longitudinal dispersion coefficient using the velocity profile in natural streams." J. Hydraul. Eng., 130(3), 227-236.

Seo, I. W., and Cheong, T. S. (1998). "Predicting longitudinal dispersion coefficient in natural streams." J. Hydraul. Eng., 124(1), 25-32.

Smart, G. M., Duncan, M. J., and Walsh, J. M. (2002). "Relatively rough flow resistance equations." J. Hydraul. Eng., 128(6), 568-578.

Taylor, G. I. (1954). "The dispersion of matter in turbulent flow through a pipe." Proc., R. Soc. Lond. A, 223(1115), 446-468.

Umeyama, M. (2005). "Reynolds stresses and velocity distributions in a wave-current coexisting environment." J. Waterway, Port, Coastal, Ocean Eng., 131(5), 203-212.

Valentine, E. M., and Wood, I. R. (1977). "Longitudinal dispersion with dead zones.” J. Hydraul. Div., 103(9), 975-990.

Vandenbruwaene, W., et al. (2011). "Flow interaction with dynamic vegetation patches: Implications for biogeomorphic evolution of a tidal landscape." J. Geophys. Res., 116, 1788-1801.

Vennard, J. K. (1961). Elementary fluid mechanics, Wiley, New York, 570. 
Copyright of Journal of Hydraulic Engineering is the property of American Society of Civil Engineers and its content may not be copied or emailed to multiple sites or posted to a listserv without the copyright holder's express written permission. However, users may print, download, or email articles for individual use. 